\section{Farbkennzeichnung der Probenbehälter (-gefäße) für Venenblut}

\section{H. Jomaa}

amedes MVZ für Laboratoriumsdiagnostik und Mikrobiologie, Halle/Leipzig GmbH, Halle, Deutschland

Englischer Begriff colour codes for single-use containers for venous blood specimen collection

Definition Unterscheidung der Probengefäße für Venenblut durch Verschluss-, Deckel-, Etiketten- oder Aufdruckfarben.

Probengefäße für Venenblut enthalten verschiedene Zusätze wie Gelpräparationen zur Stabilisierung der Barriere zwischen Serum/Plasma und Blutkuchen nach Zentrifugation, Gerinnungsaktivierung, Gerinnungshemmung (z. B. Citrat, Heparin, EDTA) und/oder zur Stabilisierung der Probe (Fluorid als Inhibitor der Glykolyse). Zur Identifizierung der Zusätze wurde eine Farbkennzeichnung der Verschlüsse und der Etiketten eingeführt. Die Farbkennzeichnungssysteme sind herstellerspezifisch.

Eine erste Normung der Farbkodierung erfolgte 1995 mit der Publikation des Standards ISO 6710:1995 durch die internatio- nale Organisation für Normung (ISO, International Organisation for Standarization). Dieses Dokument enthielt ein einheitliches Farbkennzeichnungssystem. Bei der Überarbeitung dieser ISO konnte kein Konsens mehr erreicht werden, sodass diese in der EU verworfen und durch die Europäische Norm EN 14820:2004, „Gefäße zur einmaligen Verwendung für die venöse Blutentnahme beim Menschen“, abgelöst wurde. Jedoch wurde das einheitliche Farbkennzeichnungssystem in der EN 14820:2004 nicht weitergeführt. Ähnlich wurde im Standard CLSI GP39-A6 (CLSI = Clinical and Laboratory Standards Institute) aus dem Jahr 2010 das einheitliche Farbkennzeichnungssystem verworfen. Verschiedene Versuche, einen weltweit gültigen Farbcode zu etablieren, sind bisher gescheitert.

\section{Literatur}

Europäische Norm EN 14820 v. 27.05.2004 Europ. Komitee für Normung (CEN)

Simundic AM, Cornes MP, Grankvist K, Lippi G, Nybo M, Ceriotti F, Theodorsson E, Panteghini M, on behalf of the European Federation for Clinical Chemistry and Laboratory Medicine (EFLM) (2015) Colour coding for blood collection tube closures - a call for harmonization. Clin Chem Lab Med 53:371-376 